\title{
Using Spectral Analysis to Determine the Resonant Frequency of Vibrating Wire Gages
}

\author{
HE Hu \\ China Institute of Water Resources and Hydropower Research, Beijing, 100038, China \\ Email: heehoo@qq.com
}

Keywords: Spectral Analysis; Vibrating Wire Gage; Resonant Frequency

\begin{abstract}
Vibrating wire gages have earned their well-deserved acceptance on the basis of long term stability. But it is susceptible to external electro-magnetic noise. Anti-interference ability is the main study of various detection methods. Traditionally, time-domain approaches have been used to determine the resonant frequency of those gages. This paper is proposing that spectral analysis can do a much better job. For it is greatly improved immunity to external noise, provide measurement diagnostics to help sort good data from bad, and improved measurement precision by a factor of 10 . In addition the spectral analysis method gives us useful diagnostic information, including signal amplitude, dominate competing noise frequency, signal to noise ratio and decay ratio. So using spectral analysis to determine the resonant frequency of vibrating wire gage is more advanced and applicable.
\end{abstract}

\section{Introduction}

Vibrating wire sensor is one of the most advanced sensors on measurement application at present. The output of sensor is frequency signal, which means it has lots of advantages like stable, reliable, and having little affected by electrical parameters, a small temperature drift and zero drift, and a strong anti-interference ability. The sensor can be adjusted to long term observation and long distance measurement in harsh condition [1]. Vibrating wire gauges are widely used in a variety of applications, including measuring the magnitude of various properties and forces in many construction projects, including buildings, bridges, dams, piles, tunnel linings, pipelines, anchors, and others. The gauges have been adapted to monitor stress, strain, deflection, pressure, displacement, fluid level, angular motion, and temperature [2]. These sensors have a reputation for being accurate, stable, and durable-making them well-suited for long-term static monitoring.

In conventional systems, the frequency of the vibration is calculated by measuring the average period of the vibration based on the number of zero-crossings, or number of times the wave-form crosses the point of zero amplitude, in a specified time period. While these methods are computationally efficient, they are often subject to error because the methods are unable to distinguish between wire resonance and external noise sources. Particularly, noise sources with a small amplitude and a different frequency than the vibrating wire can introduce substantial errors. Thus, a new approach is needed that is able to determine the resonant frequency of vibrating wire sensors with improved rejection of external noise sources and improved precision [3].

\section{Vibrating Wire Gages}

The internal structure of the vibrating wire gages is shown in Figure1. The gauge is constructed so that a wire is held in tension inside a small diameter, thin- walled tube that is welded or otherwise attached to a structural member. An electromagnetic coil is used to pluck or excite the wire and measure the frequency of vibration. The frequency is then used to calculate any number of the structural member's physical properties, typically by applying a series of calibration factors. The taut wire in a vibrating-wire sensor is similar to a guitar string. By increasing the tension in the wire, the wire's resonant frequency increases. While energy is coupled to a guitar string by plucking it, energy is coupled to the wire in a vibrating-wire sensor with a frequency-swept magnetic field that is included in the sensor. Once the wire is vibrating, data-acquisition 
equipment determines the resonant frequency of the wire and converts this resonant frequency into the desired engineering units. The following section describes the traditional time-domain approach used to determine the wire's resonant frequency.

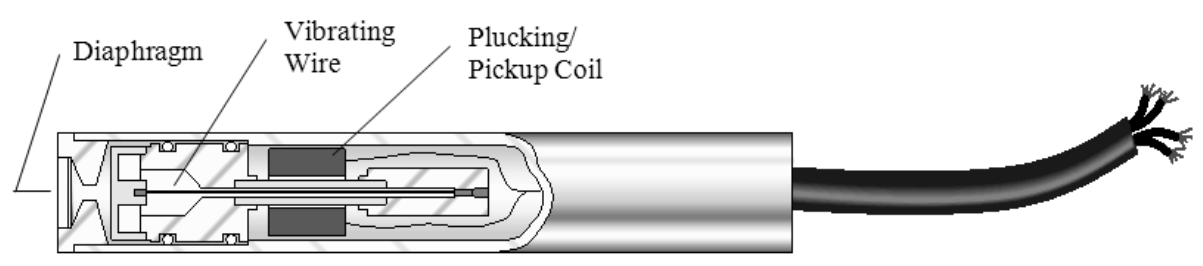

Fig.1. Cutaway of vibrating wire sensor

\section{Time-domain Analysis}

The traditional method for reading vibrating-wire sensors analyzes the response from a sensor as a function of time using period averaging. This technique excites the wire and measures the time between a predetermined number of positive-slope zero crossings in the wire's response to give the wire's resonant period (Period $=$ Time $(T) /($ Number of cycles) [4]. The resonant frequency is the reciprocal of resonant period (refer with: Figure2 (a)).

In a low-noise environment, it is an effective method of determining the resonant frequency of the wire. It has been successfully integrated into many data acquisition products that simplify its application with dedicated hardware modules, programming instructions, and software.

Figure 2 (b) displays the problem of measuring zero crossings in the presence of external noise. The external noise disrupts the signal, erroneously removing zero crossings. In general, external noise may introduce errors by adding zero crossings to, or by removing zero crossings from, the uncorrupted signal.

Even with this difficulty, engineers are sometimes able to gather useful data in noisy environments. However, this requires extra effort to eliminate noise sources or schedule measurements around periods of increased noise. An alternative approach requires data analysts to identify and discard bad data by comparing measurement data before and after noise events.

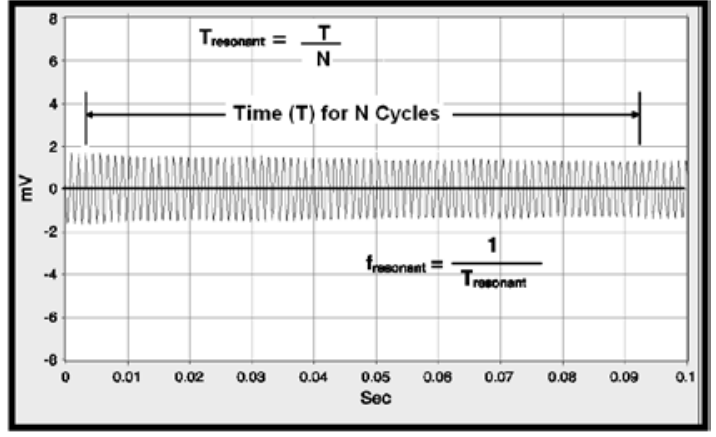

(a)

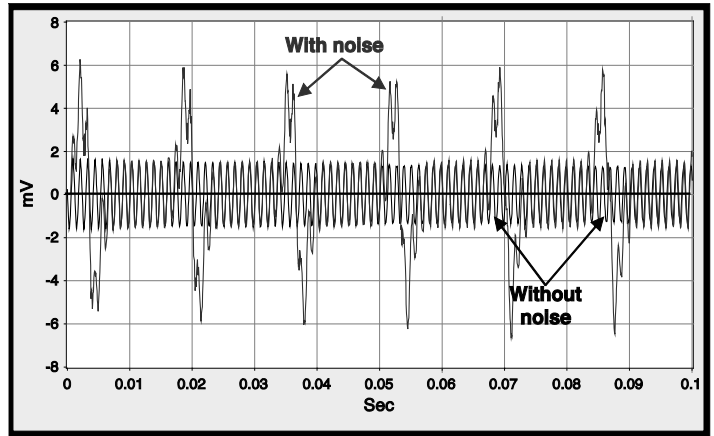

(b)

Fig.2. Previous time-domain analysis

\section{Spectral Analysis}

The spectral domain is the time domain signal after Fourier transformation. Independent variable is the frequency, the horizontal axis is the frequency, and the vertical axis is the frequency of the signal amplitude, expression of the spectral components of the signal, as shown in Figure3 (a) shows. The input signal is first passed through a variable attenuator in order to provide different measurement range. Then the signal is sampled by the low pass filter and the high frequency component is sampled. The analog signal to digital signal is converted by the high speed ADC. The final digital signal processor receives the discrete waveform data, using FFT or DFT to calculate the spectrum of the wave [5].

The spectral-analysis approach analyzes the response of the wire as a function of frequency 
(refer with: Figure 3(b)) . It makes sense to analyze the response of the wire as a function of frequency, rather than time, since we are trying to determine the resonant frequency of a tensioned wire. The new approach applies a Fourier transform with interpolation to the sampled response to move it from the time-domain to the frequency domain

Using a standard Fourier transform on a vibrating wire does not return the dominant frequency with the resolution required. This is because the total time spent measuring the wire response determines the delta $\mathrm{f}$ between spectral components in the spectrum, and a vibrating wire sensor vibrations die out fairly quickly. For instance, if we could measure the response from the wire for 0.25 seconds, the result would have a resolution of $4 \mathrm{~Hz}$. This is inadequate, as the vibrating wire manufacturers want us to resolve changes in the resonant frequency 40 times better than that, to $0.1 \mathrm{~Hz}$.

To get a result with sufficient resolution, a specialized interpolation method, developed by Richard Lyons, is required. Using this Spectral-analysis method achieves measurement precision of $0.001 \mathrm{~Hz} r m s[6]$, a significant improvement. This is about 10 times better resolution than can be achieved with the old time-domain measurement analysis.

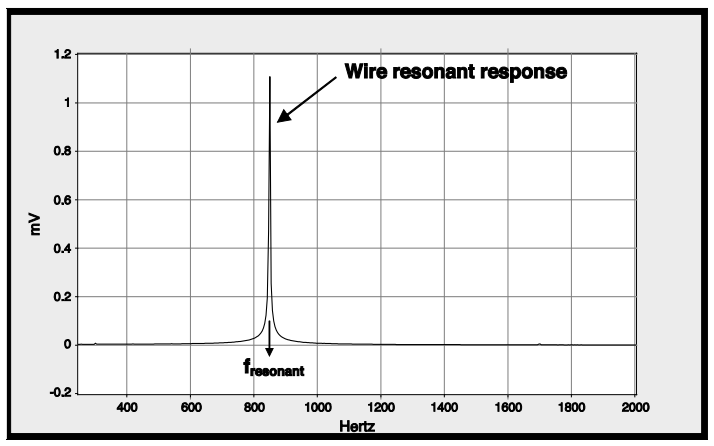

(a)

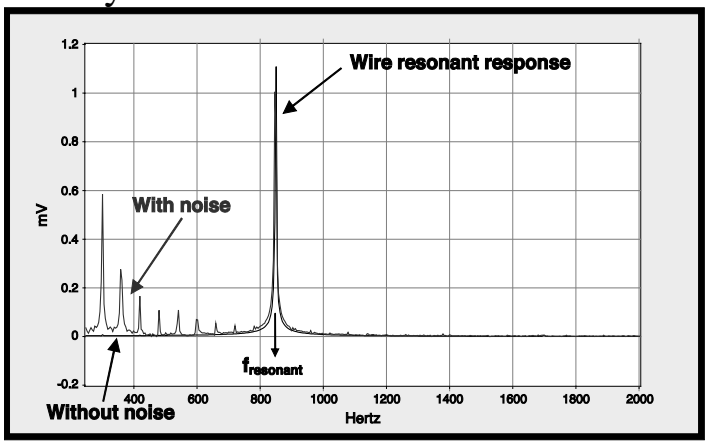

(b)

Fig.3.Frequency spectrum of vibrating wire instrument response signal

\section{Diagnostic Function of Spectral-analysis}

Spectral-analysis method can not only eliminate the noise, but also provide the signal diagnosis information, such as signal amplitude, signal to noise ratio (SNR), noise frequency and signal decay ratio, as shown in Figure4 and Fig5. These parameters are helpful to evaluate the signal quality, monitor the health status and long-term stability of the gages.

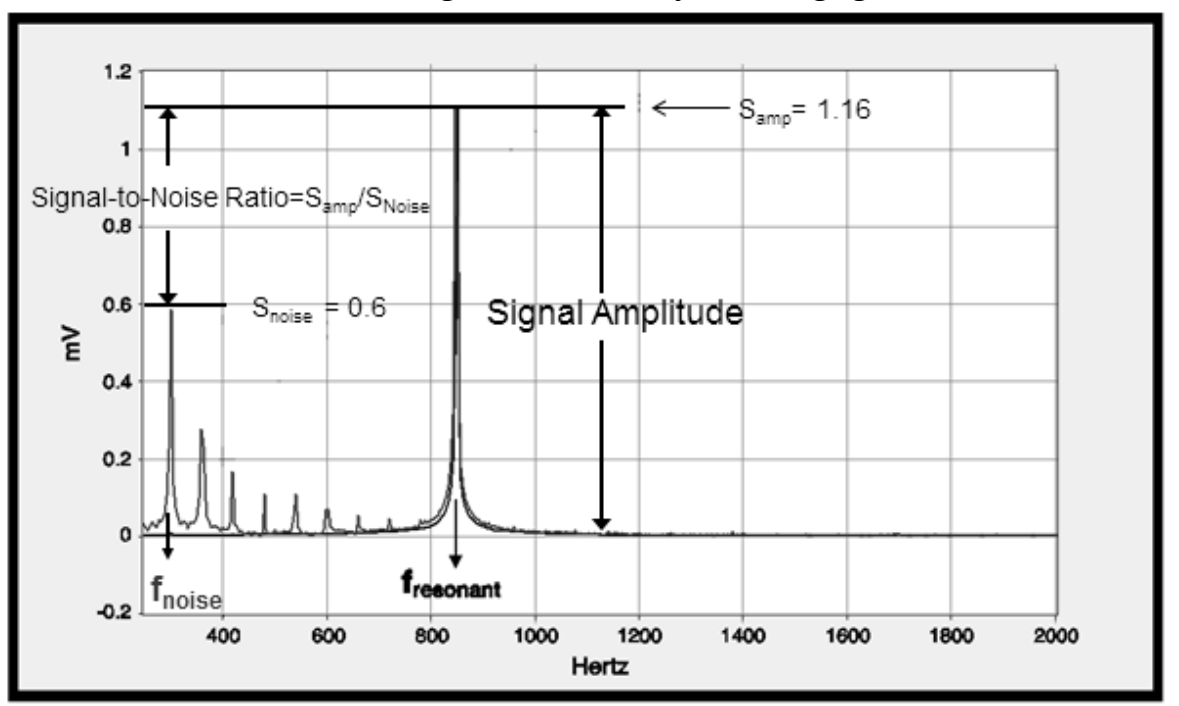

Fig.4. Diagnostic information contained in the spectrum diagram

Signal Amplitude $\left(\mathbf{S}_{\mathbf{a m p}}\right)$. The signal amplitude of the vibrating wire gages is generally RMS $1-10 \mathrm{mV}$. The long-term variation of signal amplitude can be used to monitor the health status of the sensor. 
Signal-to-noise Ratio（SNR）. It is the ratio of the amplitude of the resonant frequency signal to the signal amplitude of the maximum noise frequency. The index reflects the intensity of the effective signal to the noise signal. SNR is greater than 100 indicating that the sensor embedded environment noise interference is small.

Noise Frequency $\left(\mathbf{f}_{\text {noise }}\right)$. It can be found that the noise component of the maximum interference, and the distribution of various noise frequency is also visible, can be used to set up different types of monitoring instrument for different sampling Threshold, so as to obtain a higher SNR's resonance signal.

Decay Ratio. This is the only analysis that we perform on time series data(refer with: Figure5). Here we report how well the gage sustains its resonance after the excitation is applied. We have seen anywhere from 10 to 80 percent decay, depending on gage. As with signal amplitude, the absolute decay ratio may be of less importance than the long-term history. Vibrating wire gage manufacturers have reported that gages with poor mechanical connections on the end of the wire lose their ability to sustain this resonance.

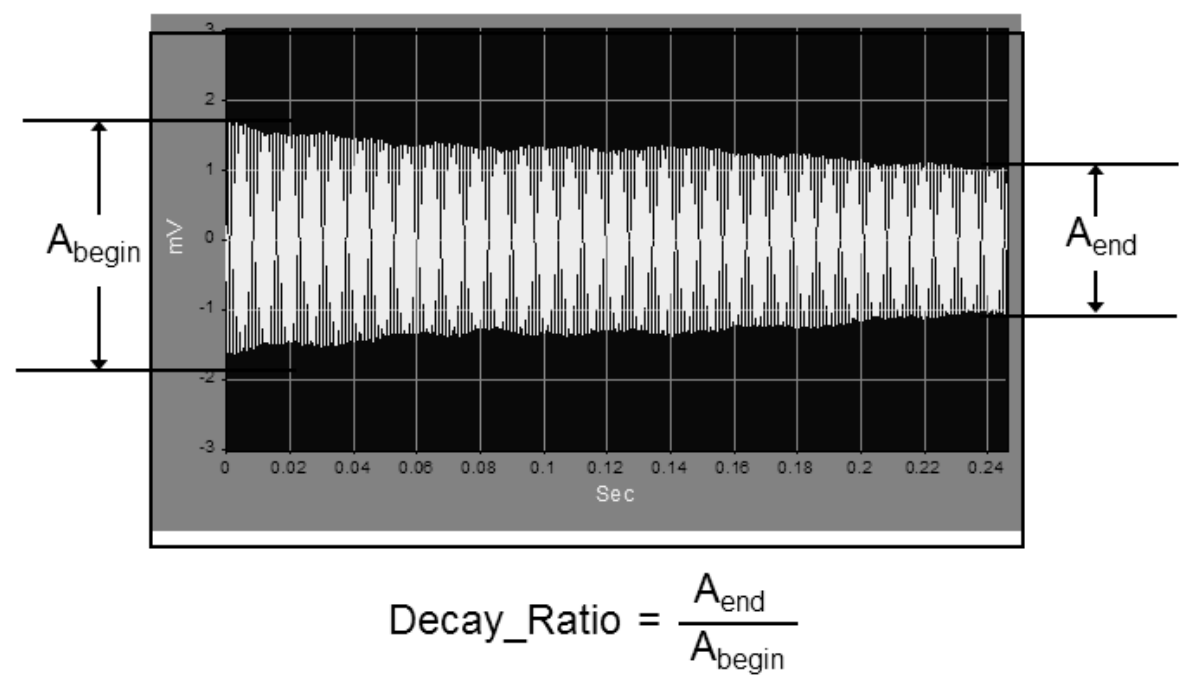

Fig.5. Timing diagram of response signal of vibrating wire instrument

These 4 evaluation criteria can be optimized by changing the sampling resolution bandwidth. When the gage(GK4000) is excited by voltage 5Vp-p and sampled with a resolution bandwidth of $100 \mathrm{~Hz}-6500 \mathrm{~Hz}$, the measured resonance frequency is $1676.02 \mathrm{~Hz}$, the signal-to-noise ratio is 8.697, the decay ratio is 0.56 , the peak amplitude is $1.61 \mathrm{mV}$, the noise frequency is $5274.78 \mathrm{~Hz}$ (refer with:Figure6); When the resolution bandwidth is changed to $1400 \mathrm{~Hz}-1800 \mathrm{~Hz}$, which is more close to the resonance frequency, the measured resonance frequency is $1676.03 \mathrm{~Hz}$, the signal to noise ratio is 7156.59 , the decay ratio is 0.59 , the peak amplitude is $2.65 \mathrm{mV}$, the noise frequency is 0(refer with:Figure7). It can be seen that the sampling resolution bandwidth is set at the narrow range of the resonance frequency, the signal to noise ratio increased by 800 times, the amplitude of the resonance signal increased by $1.05 \mathrm{mV}$, the noise is basically suppressed, the accuracy of the resonance frequency is higher and more reliable. 


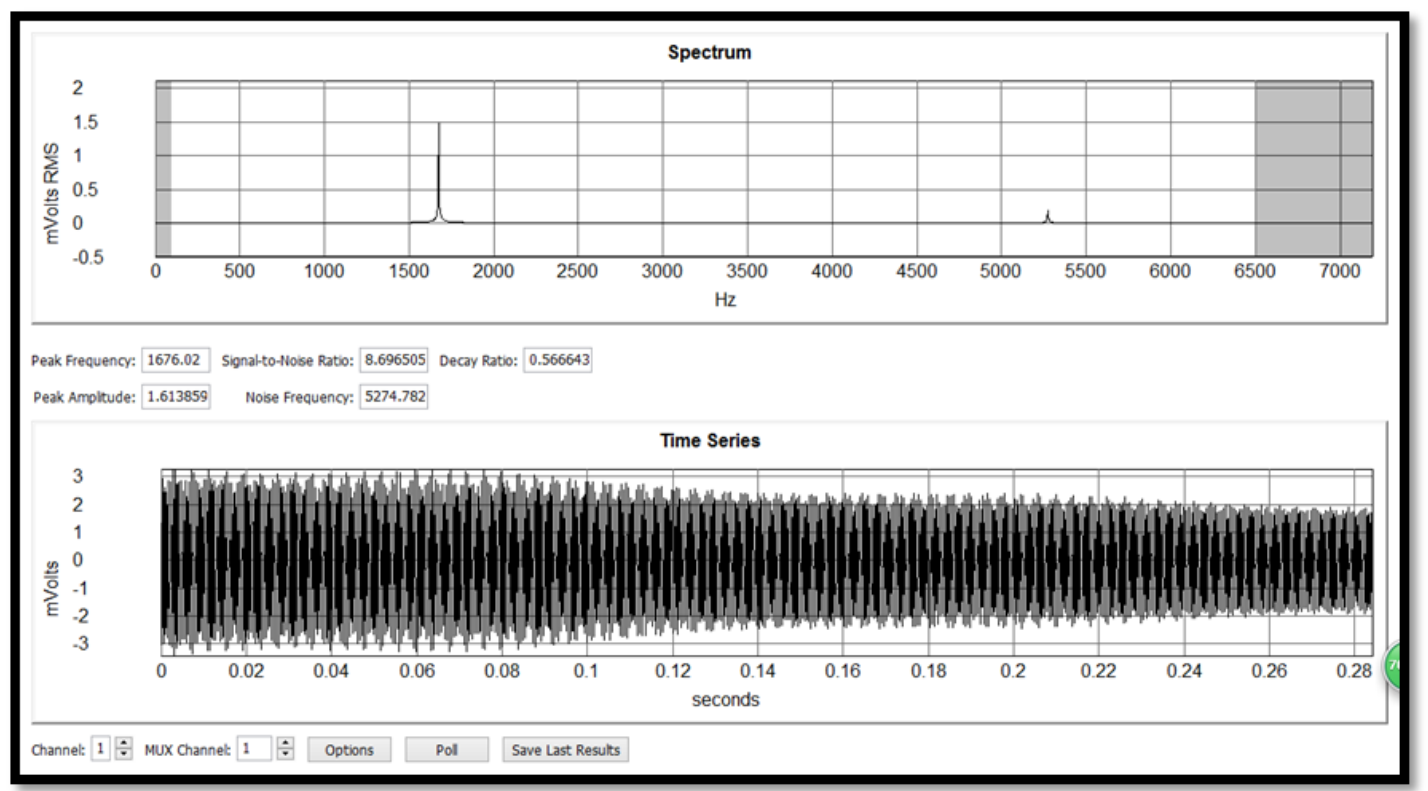

Fig.6. Spectrum and time series of GK4000 sampling signal（Bandwidth: 100Hz-6500Hz）

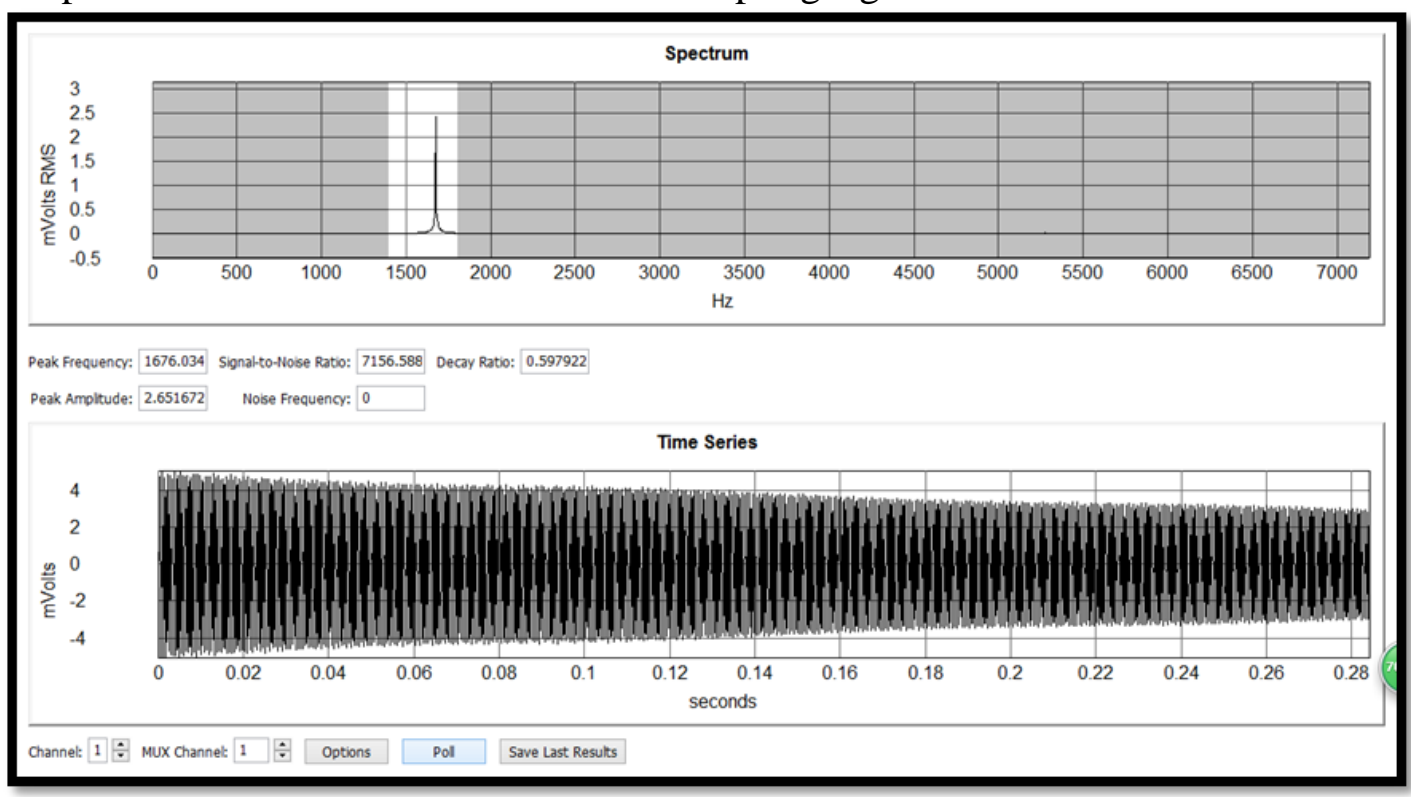

Fig.7. Spectrum and time series of GK4000 sampling signal（Bandwidth: $1400 \mathrm{~Hz}-1800 \mathrm{~Hz}$ )

\section{Conclusion}

Time domain analysis method for measuring vibrating monitoring instrument of resonance frequency, anti-interference ability is poor, cannot provide diagnostic information, measurement accuracy is higher ( $\sigma$ __resonant $=0.01 \mathrm{~Hz} \mathrm{rms}$ ). Spectral-analysis method has higher anti-interference ability, can provide multiple evaluation index for diagnosis of resonance frequency of the signal quality, has the very high measuring accuracy ( $\sigma$ __resonant $=0.001 \mathrm{~Hz}$ rms). spectral-analysis method is used to measure the resonance frequency of the vibrating wire monitoring instrument is more advanced and applicable, and it is the future research and development direction.

\section{References}

[1] GAO Yan-ying, LUAN Gui-chen, HUANG You-wen. The research and development of SF-1 type vibrating dynamometer [J]. Building science, 1998(2) 27-30. 
[2] LIU Min, SHU Nai-qiu, ZHONG Wei et al. Development of VW-1 type portable detector [J]. Chinese rural water conservancy and hydropower, 2005(1)120-124.

[3] Campbell Scientific Inc. Instruction Manual 《AVW200-series 2-Channel Vibrating Wire Spectrum Analyzer Modules》, 2012.

[4] $\mathrm{HE} \mathrm{Hu}$ and so on. High precision frequency measuring module based on MSP430 [J]. Data acquisition and processing, 2009 (24s) 241-244.

[5] KANG Bin. The principle and realization method of spectrum analysis [J]. Journal of Leshan Normal University, 2004 (12)33-35.

[6] Jacobsen et al. U.S. Patent7779690B2 (2010) 